\title{
Tres razones de la metamorfosis de las ciencias sociales en el siglo XXI
}

\author{
Three reasons for social sciences metamorphosis in the 21st century
}

Carlos Eduardo Maldonado (maldonadocarlos@unbosque.edu.co) Facultad de Medicina, Universidad EI Bosque (Bogotá, Colombia) http://orcid.org/0000-0002-9262-8879

\begin{abstract}
This paper explains the process of complexification of the social sciences during the end of the 20th century and the beginning of the 21 st century, while it provides three reasons why this has happened. The paper claims that the human and social sciences must undergo a radical metamorphosis if they want to keep alive at all. Even though, nothing obliges that these sciences must go on existing. At the end, as an open-ended conclusion, it is pointed out how, because of the changes that express a deep crisis, how a brand-new science of the impossible has emerged.
\end{abstract}

Key words: social sciences, history of science, epistemology, complexity, impossibility.

\section{Resumen}

Este artículo explica la complejización de las ciencias sociales en el curso del siglo XXI, y aporta tres razones por las que este proceso ha tenido lugar. La tesis que se defiende aquí es que las ciencias sociales sufren una metamorfosis radical que deben asumir si quieren seguir existiendo. Aunque nada obliga a que tengan que seguir existiendo. Al final, en unas concusiones abiertas, se señala cómo, como consecuencia de las transformaciones en crisis, emerge una ciencia de lo imposible.

Palabras clave: ciencias sociales, historia de la ciencia, epistemología, complejidad, imposibilidad.

\section{Introducción}

Es suficientemente sabido: las ciencias sociales nacen como el proyecto de una clase social triunfante que necesitaba explicarse y comprenderse a sí misma, y a lo diferente y lo otro con respecto a si misma. Análogamente, por ejemplo, a la novela, un invento del siglo XVIII-XIX. Este texto se divide en dos partes. De un lado, el estudio, breve, de cómo el destino de las ciencias sociales se está jugado. Y con él, pareciera, también el destino de una buena parte del mundo. A la manera de los mejores mitos, el nacimiento de las ciencias sociales marcó su destino. Hasta que se hizo posible una metamorfosis de las mismas. De otra parte, la sección principal, se trata de entender las razones de las metamorfosis de las ciencias sociales. De esta suerte, aquella parte es el pretexto para entender esta otra, pero sirve al mismo tiempo como su condición.

Este texto defiende una tesis, a saber, las ciencias sociales deben metamorfosearse si quieren seguir existiendo. En efecto, lo que más ha habido en la historia de la ciencia y la cultura es una enorme mortandad de conceptos, ciencias y disciplinas. La lista sería larga e incluye al flogisto, la alquimia, el éter, 
la fisiognómica, los espíritus animales y los homúnculos, la teología como scientia magna, la propia filosofía como via regia, y muchas más.

Vivimos una crisis sistémica y sistemática a la vez. Frente a esta crisis, pareciera haber una atmósfera de descrédito hacia la ciencia, y muy en primer lugar hacia las ciencias sociales, y también un descrédito de la razón y la racionalidad en general (Horgan. El fin de la ciencia, Nudler. La racionalidad). La importancia en general de las ciencias sociales estriba en que ellas nos dicen cómo hemos vivido y cómo podemos vivir, en toda la extensión de la palabra -con referencias directas a los planos político, económico, sociológico, psicológico y otros. El mundo está sobrediasgnosticado en sus crisis. Pero parecieran no haber salidas. Un espíritu derrotista marca la obra de numerosos e importantes autores de las ciencias sociales. La lista de autores derrotistas es casi innumerable e incluye a Bauman, Morin, Popper, Byung-Chul Han, Derrida, Rorty, Habermas, Rawls, En el pasado reciente forman parte también de esta línea pesimista Freud, Husserl, Heidegger, Lévinas, Spengler, Foucault, Bordieu y Gore. Estos son algunos de los nombres de una posición que puede denominarse sin ambages como "milenarista" (Cohn. En pos del milenio). En cualquier caso, social, política y culturalmente, el neoliberalismo es una forma de vida pesimista.

Este artículo no pretende, en absoluto, elaborar una lista de motivos. Más exactamente, pretende aportar algunas explicaciones de lo que ha sucedido y está aconteciendo en el mundo científico en general, y en el de las ciencias sociales, en particular. La mirada se orienta hacia el presente inmediato y hacia el futuro, pero ello toma impulso de algunos procesos nucleares en el pasado.

\section{Primera razón: el mundo cambió}

De manera muy significativa, el nacimiento de las ciencias sociales es contemporáneo con la conformación de los estados-nación en Europa y alrededor del mundo. Pues bien, de manera muy precisa, la historia subsiguiente es exactamente el resultado de la idea y la existencia del estado nación: la primera y la segunda guerra mundial, las guerras de Corea, Vietnam, Irak I, Irak II, Siria, para mencionar solo las mayores. En otra latitud, es toda la historia del neo-colonialismo y la serie de invasiones a países de América Latina (sistemáticamente desde 1846 hasta el 2002, cuarenta y ocho en total). Posteriormente, superponiéndose a los nacionalismos, aparece el poder corporativo (ocho transnacionales de la alimentación a escala global, un pequeño pull de medios masivos de comunicación y de casas editoriales, etc.). Y siempre el complejo industrial-militar, una nueva realidad, cuando se lo mira con los ojos del pasado.

Cuando nacen las ciencias sociales y humanas vivíamos un mundo de suma cero, con todo lo que ello implica. Hoy, por el contrario, vivimos un diferente suma cero, en el que, por consiguiente, hay múltiples entrelazamientos, sensibilidades recíprocas, interdependencias en prácticamente todos los órdenes. La mariposa de Lorenza revolotea por todo el mundo, la política es hoy geopolítica, y la economía depende de decisiones corporativas tanto como de los microclimas, por ejemplo.

Las ciencias sociales y humanas se ocupan de sistemas sociales humanos, una afirmación que tendría todas las luces de pleonasmo, si no fuera por el desarrollo de estas ciencias en el siglo XXI. Originariamente, las ciencias sociales son ciencias antropológicas y antropocéntricas. Sin embargo, con el devenir el tiempo hasta descubrimos que en realidad existen tres clases de sistemas sociales: sistemas sociales humanos, sistemas sociales naturales y sistemas sociales artificiales. Lo humano como tal se complejiza. Pues bien, entre el siglo XIX y el presente aprendimos la profunda crisis medioambiental como nunca antes ninguna época lo había presenciado (cfr. primero y segundo informes al Club de Roma 1971-1977, el cuarto informe 
elaborado por von Weizsäcker de 1996, entre otros). Sin la menor duda, el más difícil de todos los problemas es hoy por hoy la crisis del medioambiente y lo es porque es inseparable de otras crisis del mundo moderno.

De manera puntual, la crisis del medioambiente pone de manifiesto un hecho sin igual en la historia de la humanidad. Mientras que tradicionalmente el input y el output coincidían: en el ser humano, hoy, por primera vez, el input y el output son diferentes. En aquella interpretación, son los seres humanos quienes actúan y toman decisiones, en función del propio bienestar e interés humano. En este otro caos, por el contrario, el input son los seres humanos, pero el output es la naturaleza. Así, el antropocentrismo en toda la extensión de la palabra queda desplazado en favor de, llamémoslo, un ecocentrismo o biocentrismo.

De consuno, varias ciencias y disciplinas emergieron que contribuyeron a transformar radicalmente la imagen en la que surgieron y vivieron durante mucho tiempo las ciencias sociales. Cabe destacar a la etología (Lorenz, Tinbergen, de Waals), la endosimbiosis (Margulis) y la epigenética (Waddington, Jablonka y Lamb). Por su parte, la biología en general, y en particular la biología de sistemas, ha puesto de manifiesto que los seres humanos son holobiontes, esto es, bastante más naturaleza, que otra cosa. De esta suerte, la imagen clásica del ser humano como el epicentro o el cénit de la creación estalló en mil pedazos, dando lugar a una visión más amplia, incluyente, en la que las jerarquías y las centralidades en el universo y en la naturaleza se revelaron como pseudo-problemas. En este marco, aprendimos la idea de que vivimos una era secular (Taylor. A secular age), en la que nuevas formas de ética y más exactamente un pluralismo ético y axiológico fueron reconocidos y tematizados ampliamente. En el espectro humano, hemos llegado a aprender la idea de un pluralismo de humanismos, sin que ninguna pueda erigirse como preferencial o superior a cualquier otro. Manifiestamente, el mundo ha terminado por volverse crecientemente complejo.

En el tiempo transcurrido entre mediados del siglo XIX y el presente tuvieron lugar numerosos eventos totalmente inesperados en su momento: la revolución rusa de 1917, el martes negro de 1929, el ascenso al poder de Hitler, la conformación de dos bloques rígidos en medio de la guerra fría, explosiones de centrales nucleares en Estados Unidos y en la Unión Soviética, la revolución cubana, la rebelión de 1968 en Mayo y, en general, todos los movimientos sociales y políticos en los años 60, el surgimiento de la teología de la liberación, la crisis energética de 1977, la caída del Muro de Berlín, la crisis económica de las empresas .com alrededor de finales del siglo XX, el Brexit, la irrupción de la China como una gran potencia actualmente y verosímilmente como la primera potencia mundial en todos los órdenes en el futuro inmediato, por mencionar tan sólo algunos de los más significativos.

Concomitantemente, la ciencia aprendió en general nuevos conceptos, teorías y disciplinas que correspondieran a este mundo. Entre estas cabe destacar, la teoría de perturbaciones, la teoría de fluctuaciones, la incertidumbre (Heisenberg) y en general la física cuántica (Forman), la teoría de sistemas (Von Bertallanfy y otros), las sinergias (Haken), el caos (Lorenz), las catástrofes (Thom), la complejidad (Weaver, Kolmogorov, Chaitin), los equilibrios dinámicos (Prigogine), las redes complejas (Barabasi, Strogatz), la percolación (Grimmett), la inteligencia de enjambre (Dorigo, Beaunabau), la topología (Smale), la cohomología (Penrose), por ejemplo. En todos los casos se trató del proceso mediante el cual se acuñan conceptos, categorías y metáforas a fin de poder explicar un mundo en general que ya no existía en el equilibrio. Antes, por el contrario, se trata de un mundo marcado por procesos, no ya por estados. Dicho sucintamente, y a ello apunta exactamente la idea de un mundo diferente de suma cero, el mundo general aprendió la no-linealidad. Y debimos aprender a vivir con ella. Las ciencias sociales y humanas no fueron ajenas a estas dinámicas, fenómenos y comportamientos. 
Como sostienen Prigogine y Stengers: "es alrededor de los temas de irreversibilidad, del proceso de organización y de la innovación, donde se han desarrollado las teorías que nos permiten hoy hablar de una metamorfosis de la ciencia" (Prigogine y Stengers 2002:60).

\section{Segunda razón: aparecen nuevas formas de ver, de explicar, por tanto, nuevos métodos y nuevas técnicas}

Cuando aparecen nuevos fenómenos, nuevos comportamientos o nuevos sistemas, se configuran, concomitantemente, nuevos lenguajes que permiten hacerlos visibles y explicarlos. La historia de la humanidad ha estado indudablemente vinculada a la historia misma de la escritura, en el sentido más amplio de la palabra. En el espectro humano social, la escritura aparece como la memoria de la palabra hablada. Sin ambages, esta memoria ha sufrido dos revoluciones: la primera, con la invención de la imprenta (verosímilmente gracias a Guttenberg en el siglo XV), y luego con la aparición de internet, en los años 1980.

De manera puntual, internet llegó para transformar la forma como vemos el mundo y la realidad, y como los seres humanos se relacionan consigo mismos y con el entorno. Las metamorfosis de las ciencias sociales en el curso del siglo XX y XXI son indudablemente el resultado de la llegada de internet. Más exactamente, se trata de la aparición de la web 1.0, 2.0, 3.0 y la discusión en curso acerca de la web 4.0 y la web 5.0 (la web emocional). Tres instancias resultan determinantes en este proceso: el aprendizaje de máquinas (machine learning), el aprendizaje profundo (deep learning) y los sistemas expertos. Todo ello apunta hacia una dúplice dirección: la inteligencia artificial y la vida artificial. En esta historia, el surgimiento de la web profunda (deep web) termina por configurar un escenario de máxima complejidad informacional. En la base de esta historia se encuentra, manifiestamente, el papel del computador y de la computación. Dicho de manera puntual, el computador es una herramienta conceptual: tiene memoria, tiene sintaxis, tiene semántica, aprende por sí mismo, y cuando no está prisionero de la carcasa (hardware), existe en el mundo como robótica, a saber, como robótica y robótica de enjambre.

Pues bien, en el plano de la sociedad en el sentido primero de la palabra, en el lapso que transcurre entre el texto fundacional de A. Compte y hoy, aparecen las cuatro revoluciones industriales, con todo lo que ello implica, y la ciencia clásica conoce la segunda revolución científica (la teoría cuántica) y la tercera revolución científica (las ciencias de la información y el procesamiento de la información). Una revolución industrial es en general una revolución en el trabajo y en la forma de organización del trabajo. Cabe decir que las ciencias sociales clásicas (esto es, aquellas eminente y distintivamente antropológicas y antropocéntricas), son prácticamente contemporáneas con la primera y la segunda revolución industrial. La tercera revolución industrial aparece en el año 2011 y la cuarta revolución es anunciada originariamente en el 2016. Como se aprecia, y este constituye un motivo determinante de la segunda razón de la metamorfosis de las ciencias sociales, el ritmo de desarrollo del conocimiento ha llegado a ser, más que exponencial, hiperbólico. La interfaz sociedad-tecnología al mismo tiempo responde a este ritmo hiperbólico y contribuye a catapultarlo.

Metodológicamente, uno de los conceptos más importantes cuando han nacido las primeras ciencias sociales es la "variable"; hoy, por el contrario, es la idea de datos y grandes bases de datos. Un cambio absolutamente sin igual. De esta suerte, los métodos y las técnicas para ver el mundo y la realidad se transforman radicalmente, y aparecen así el modelamiento y la simulación. Todo parece constituir una unidad compleja y dinámica. En otras palabras, las viejas metodologías, basadas en observación, descripción, formulación de hipótesis y demás se hacen al cabo vetustas e insuficientes para comprender 
la complejidad de la realidad en general. La distinción entre métodos cualitativos y métodos cuantitativos corresponde, en rigor, a la primera revolución científica; esto es, a la ciencia clásica o la ciencia de la modernidad. Lo mismo acontece con la idea de métodos mixtos o híbridos.

El computador en general, la computación e internet y las web constituyen memorias de las palabras, de los actos y los gestos. Reflejan la vida del mundo y contribuyen a su vez a esculpir la faz misma de las sociedades y los individuos. La grafía ya no es la única o la principal forma de comunicación, y tampoco constituye la principal forma de memoria de las palabras, los actos y las vidas. Existen hoy por hoy muchas formas de expresión; las redes sociales y numerosas aplicaciones son la mejor forma como aparece la sociedad y el mundo ante sí mismos. A esto habría que agregar YouTube y varios otros canales. Todo lo anterior se concentra en un título genérico, a saber: la inteligencia artificial. Ciertamente ante el panorama anterior existen voces temerosas y conservadores, críticas de la vida ante las pantallas, tanto como voces optimistas y emancipadoras que reconocen que el conocimiento, por primera vez, es un bien de la humanidad y no pertenece a nadie. Las ciencias sociales son simplemente el terreno de batalla entre unos y otros. Como quiera que sea, como se aprecia sin dificultad, una segunda razón de la metamorfosis de las ciencias sociales son las nuevas formas emergentes y que a la fecha se materializan como la tercera y la cuarta revolución industrial, que introducen nuevas herramientas, nuevos métodos, nuevos lenguajes, nuevas estructuras, nuevas visiones y formas y estilos de vida. Las ciencias sociales y humanas tradicionales no podían saber nada de esto. Pero hoy deben poder aprenderlo.

La importancia de las tecnologías, en toda la acepción de la palabra -tecnologías convergentes: NBIC+S: las nanotecnologías, la biotecnología, las tecnologías de la información, las tecnologías del conocimiento y la dimensión social de la tecnología-, representa una verdadera inflexión en el conocimiento con numerosas aristas y derivaciones. No en última instancia, "nuestro poder para intervenir en nuestra propia evolución es, posiblemente, la mayor de las innovaciones de todos los tiempos, el mayor de los dilemas, porque es también, al menos en teoría, la mayor de las oportunidades" (Watson 2017:457).

En síntesis, dicho puntualmente, el computador y la computación, de un lado y, de otra parte, pero estrechamente vinculada a la anterior, la web, configuran realidades al mismo tiempo culturales, científicas y metodológicas que impactan ampliamente la vida de las sociedades y de los individuos, acaso, análogamente, como en su momento lo hizo la escritura primero y la imprenta luego. Las ciencias sociales y humanas no pueden abstraerse de estas nuevas estructuras y dinámicas. Aunque la realidad es que permanecen aún lejanas a la computación y a la web, en el sentido más preciso y fuerte de la palabra.

\section{Tercera razón: cambió el ecosistema de las ciencias y el conocimiento}

Concomitante con las dos razones anteriores, la tercera razón tiene que ver con un hecho de una radicalidad aún mayor. Se trata de que el ecosistema entero del conocimiento, esto es, de las ciencias y disciplinas cambió profundamente. Quisiera detenerme en dos hechos contundentes de este cambio del ecosistema del conocimiento. De una parte, de manera significativa, se trata del Informe de la Comisión Gulbenkian, dirigido directamente a las ciencias sociales, consistente en el llamado a abrir las ciencias sociales. A primera vista, se trata de un llamado poco comprensible. En efecto, las ciencias sociales siempre han estado abiertas al mundo, a la sociedad, a la realidad. La forma más puntual como fueron llamadas muy pronto luego de su nacimiento fue como una "física social". De manera atávica, la mayoría de las ciencias sociales hablan y trabajan con la idea de "trabajo de campo", "pasantías", "salidas de campo" y otras expresiones similares. Si es así, abrirse al mundo sería una idea redundante e incluso trivial. 
Pues bien, una mirada cuidadosa al Informe pone de manifiesto que, por el contrario, y de manera fundamental, se trata de la invitación a las ciencias sociales a que se abran... al otro grupo de ciencias, las ciencias naturales, físicas y exactas, para decirlo con el lenguaje propio del siglo XIX. De hecho, la verdadera interdisciplinariedad no sucede cuando hay diálogo de ciencias y disciplinas al interior de una misma familia, sino, por el contrario, cuando sucede entre familias distintas: las ingenierías, las ciencias naturales, las ciencias sociales, las humanidades. Excepto por alguna aproximación, por lo demás, superficial o artificiosa por parte de la economía, las ciencias sociales y humanas han sido distintivamente cualitativas y anumericas. El aparato matemático de las ciencias sociales, por ejemplo, es escaso, si es que acaso llega a estar presente.

En estrecha relación con lo anterior, el otro elemento determinante del cambio del ecosistema del conocimiento consiste en la aparición y la distinción entre la gran ciencia y la pequeña ciencia (de Solla Price. Little Science, Big Science... and Beyond). En verdad, contra toda apariencia, que haría pensar acaso en la aparición de nuevas ciencias y disciplinas sociales y mixtas en el marco de las ciencias sociales en el transcurso del siglo XX y lo que va corrido del siglo XXI, la configuración de la gran ciencia y la pequeña ciencia marca asimetrías profundas, y acaso irreconciliables. La gran ciencia se caracteriza por que funda programas de investigación y trabaja en torno a programas de investigación. En contraste, la pequeña ciencia se organiza alrededor de grupos y líneas de investigación. La gran ciencia es ciencia que compete e interesa por igual a científicos, ingenieros, humanistas, tomadores de decisión, militares, financistas, administradores y a sectores de la sociedad civil. La pequeña ciencia, por el contrario, interesa tan solo a los investigadores de cada área o experticia. Enormes equipos humanos, tecnológicos, financieros y logísticos sostienen y catapultan a la vez a la gran ciencia y muy importantes recursos de energía, económicos y políticos pivotan en torno a la gran ciencia. Literalmente, miles de investigadores constituyen y definen a la gran ciencia (en su mejor momento, por ejemplo, el proyecto Manhattan reunía alrededor de 5000 investigadores, la inmensa mayoría con Ph.D; el CERN, por ejemplo, aloja permanentemente a alrededor de 2500 investigadores, entre físicos, químicos, matemáticos, ingenieros, entre otros, la gran mayoría también con título de doctorado, además de aquellos investigadores itinerantes o a distancia que colaboran con el CERN). De manera distintiva, la gran ciencia trabaja fuertemente en redes de investigación, a escala nacional, continental y mundial. Dicho de manera puntual, algunos ejemplos de la gran ciencia son el Proyecto Manhattan, el CERN, el Proyecto Genoma Humano, el Proyecto y la Iniciativa BRAIN, la NASA, la Agencia Espacial Europea, la exploración y conquista del espacio extraterrestre, y varias otras. La tabla 1 sintetiza la asimetría entre la pequeña ciencia y la gran ciencia.

Tabla 1. La asimetría grande entre la gran ciencia y la pequeña ciencia

\begin{tabular}{|l|l|}
\hline Gran ciencia & Pequeña ciencia \\
\hline Programas de investigación & Líneas y grupos de investigación \\
\hline Investigación básica & Investigación aplicada y experimental \\
\hline
\end{tabular}

Fuente: Elaboración propia.

En la base de la tabla 1 se podrían indicar algunos de los problemas fundamentales que, originándose en los terrenos de las ciencias sociales y humanas, podrían ser incluidos dentro de la gran ciencia: los problemas medioambientales, sociales y de salud (o médicos). En cualquier caso, quisiera decirlo de manera puntual y directa: las ciencias mal llamadas duras integran, grosso modo, a la gran ciencia. Por su parte, en su conjunto, las ciencias sociales siguen formando parte de la pequeña ciencia. Como se aprecia, la tercera razón de la metamorfosis de las ciencias sociales en el siglo XXI plantea un enorme reto de orden epistemológico, metodológico y organizacional para las ciencias sociales y humanas. 


\section{Sintetizando: ¿Hacia dónde se produce la metamorfosis?}

Como se aprecia sin dificultad, asistimos hoy por hoy a una complejización de las ciencias sociales que ya nada tienen que ver con el programa Comptiano. Literalmente, las ciencias sociales y humanas han dejado de ser exclusiva y distintivamente humanas y sociales-humanas. Han debido aprender otras tradiciones, otros lenguajes, otras metodologías. Ha habido transformaciones profundas al interior de varias de las ciencias sociales y humanas; asimismo, se han modificado las relaciones, de vecindazgo, digamos, si cabe, con otras ciencias. El mundo cambió, por definición, de manera impredecible. Y ulteriormente todo el ecosistema del conocimiento se transformó, modificando ulteriormente la forma como la sociedad se ve a sí misma y se hace posible a sí misma. El nombre puntual de este cambio son las revoluciones industriales. Sin embargo, es suficientemente sabido que éstas se deben a transformaciones en los planos de las técnicas y las tecnologías, tanto como de la producción y el corpus científico y de investigación. No en última instancia, tanto el aparato productivo, como el propio trabajo y el sistema de educación se encuentran a la vez atravesados por estos procesos y son una de las fuentes de los mismos.

Digámoslo de manera directa y puntual. La razón por la que las ciencias sociales han debido transformarse radicalmente es que han tenido que hacerlo si quieren seguir existiendo. Solo que existirán, sugerimos, con otros nombres, con otras expresiones, en otras dimensiones. Nada obliga a las ciencias sociales y humanas a que deban seguir existiendo. Al fin y al cabo, lo que más habido en la historia de la civilización humana, análogamente a la evolución de la naturaleza, es una enorme mortandad de ciencias, disciplinas, conceptos, categorías. Desde la fisiognómica hasta el flogisto, desde la teología como ciencia magna al éter; la lista sería verdaderamente amplia. Nuevas ciencias sociales están emergiendo que no tienen absolutamente nada que ver con aquellas que surgieron en el curso del siglo XIX y comienzos del siglo XX. Pues bien, la metamorfosis no se produce al interior de una sola, cualquiera, de las ciencias sociales $y$ humanas. Tampoco se trata de un cambio en algunas de ellas. Más radicalmente, se trata de una transformación genética, por así decirlo, que modifica a todos los integrantes de las ciencias humanas y sociales. Un cambio en el genotipo, y no solamente en el fenotipo (Kaku. Physics of the impossible). Emergen, en consecuencia, nuevas ciencias sociales y humanas, que incluso ya no se llaman de esa manera. Se trata de las ciencias sociales complejas (complejidad de las ciencias sociales), las ciencias sociales cuánticas, las neurociencias sociales, las ciencias sociales interdisciplinarias, las ciencias sociales computacionales, las ciencias sociales del no-equilibrio, en fin, la ciencia social generativa.

En síntesis, en palabras de Couloubaritsis: "Lo que explica la estructura contemporánea de la técnicoeconomía en tanto que estructura histórica que se constituye y se manifiesta a través de innumerables configuraciones es bien el fondo de libertad que fue lo que hizo posible su realización y que favoreció su efectividad (...). Y es precisamente este fondo de libertad que le confiere a cada quien el poder de hacer tanto el bien como el mal lo que explica tanto sus logros para la humanidad (...) como sus distorsiones maléficas" (Couloubaritsis 2014:658).

\section{Conclusiones abiertas}

Las ciencias sociales se complejizan, debido a la complejización misma del mundo y de la realidad. La complejización de las ciencias sociales significa exactamente que han dejado de pensar en términos de causalidad, en cualquiera de sus variantes, que han aprendido la no-linealidad, en fin, que han aprendido de la incertidumbre del mundo y la realidad, y que han aprendido, ulteriormente, nuevos métodos, aproximaciones, lenguajes y experiencias de ciencias a las que nunca estuvieron efectivamente abiertas. Frente a los avatares del mundo y la naturaleza, la ciencia de punta ha ampliado de manera antes 
inimaginable su espectro. La buena ciencia ya no piensa ni estudia única o principalmente lo real, lo que hay, lo que sucede, lo que está allí. Además, ha aprendido a pensar en lo posible. Y es sabido que las matemáticas son exactamente el trabajo con espacios de posibilidades.

Tabla 2. Modos de ciencia de lo imposible

\begin{tabular}{|c|c|c|}
\hline Origen & Modo & Autores \\
\hline Matemáticas, lógica y filosofía & $\begin{array}{l}\text { Paradojas, como la del hotel } \\
\text { infinito de Hilbert, o el abuelo } \\
\text { y la máquina de viajar en el } \\
\text { tiempo, o de Russell }\end{array}$ & $\begin{array}{l}\text { D. Hilbert, Peano, B. Russell, y } \\
\text { muchos más }\end{array}$ \\
\hline Lógica & $\begin{array}{l}\text { Teorema de la incompletitud } \\
\text { de Gödel }\end{array}$ & K. Gödel \\
\hline Física cuántica & $\begin{array}{l}\text { La incertidumbre de } \\
\text { Heisenberg }\end{array}$ & W. Heisenberg \\
\hline Economía & $\begin{array}{l}\text { Teorema de imposibilidad de } \\
\text { Arrow }\end{array}$ & K. Arrow \\
\hline $\begin{array}{l}\text { Matemáticas, topología, teoría } \\
\text { de homologías }\end{array}$ & Cohomología & $\begin{array}{l}\text { Kolmogorov, S. Smale, R. } \\
\text { Penrose }\end{array}$ \\
\hline $\begin{array}{l}\text { Teoría de las probabilidades, } \\
\text { estadística, complejidad }\end{array}$ & Cisnes negros & N. Taleb \\
\hline $\begin{array}{l}\text { Teoría de la complejidad } \\
\text { computacional }\end{array}$ & $\begin{array}{l}\text { Problemas indecidibles, en el } \\
\text { marco de la teoría de la } \\
\text { complejidad computacional }\end{array}$ & Turing, Karp, Cook y Levin \\
\hline $\begin{array}{l}\text { Teoría de la computación, } \\
\text { hipercomputación }\end{array}$ & $\begin{array}{l}\text { Cualquier máquina que no } \\
\text { obedezca la tesis Church- } \\
\text { Turing }\end{array}$ & $\begin{array}{l}\text { A. Church, A. Turing, } \\
\text { Syropoulos }\end{array}$ \\
\hline $\begin{array}{l}\text { Ciencias de la complejidad, } \\
\text { teoría de la computación }\end{array}$ & Hipercomputación biológica & $\begin{array}{l}\text { M Burgin, S. Cooper, } \\
\text { Maldonado y Gómez-Cruz }\end{array}$ \\
\hline $\begin{array}{l}\text { Matemáticas, estadística, } \\
\text { teoría del valor extremo }\end{array}$ & $\begin{array}{l}\text { La transición de eventos raros } \\
\text { a eventos imposibles }\end{array}$ & $\begin{array}{l}\text { G. King and L. Zheng, } \\
\text { Maldonado }\end{array}$ \\
\hline
\end{tabular}

Fuente: Elaboración propia.

Queremos sostener que los cambios expresados en las tres razones anteriormente mencionadas han producido un giro sin igual en la ciencia en general. La buena ciencia de punta ya no piensa única ni principalmente lo que sucede, lo que hay, lo que es, lo real (como se lo quiera denominar). Además, y fundamentalmente la buena investigación de punta se ocupa (cada vez más) por lo posible. Esto es, por los desarrollos probables, hipotéticos y contingentes de lo que hay, lo que sucede, lo que está. Pues bien, como resultado de la complejización del mundo y de la ciencia en general más radicalmente aun, la ciencia ha comenzado a aprender a pensar lo imposible. Esto es, lo que anteriormente era inimaginado, inaudito, inverosímil. Sin la menor duda, la simulación ha hecho una contribución importante al respecto. Lo que otrora pareciera inimaginable, inopinado, inaudito, se revela ahora como posible e incluso como necesario. Pensar lo imposible y en ocasiones trabajar para que sea efectivamente posible, es más, bastante más que simplemente pensar en utopías.

Quisiera terminar empleando una analogía que distingue entre cine de industria y cine de autor. El cine de autor termina siempre en punta: open-ended conclusion. Se trata de ilustrar, primero, que existe hoy por hoy, como resultado de la transformación del ecosistema de las ciencias, genéricamente, una ciencia de 
lo imposible; no ya solamente de lo real y de lo posible. La tabla 2 sintetiza los modos de la ciencia lo imposible, señalando los orígenes, los modos y algunos de los autores que se encuentran en el origen de las ciencias de lo imposible. Quisiera decirlo a manera de conclusión: una ciencia de lo imposible es el resultado de la profunda transformación de las ciencias sociales y humanas.

A título ilustrativo, las ciencias sociales han debido explicar fenómenos que eran imposibles hasta que sucedieron: el martes negro de 1929, el ascenso al poder por parte de Hitler en 1933, la caída del murto de Berlín en 1989, las explosiones de las centrales nucleares de Three Mile Island de 1979 y Chernobil en 1986, la elección de jesuita argentino como Papa, Francisco, y muchos otros más. Lo imposible ha aparecido en la historia de la ciencia, en ocasiones, como posible, y a veces, como real efectivamente. Sin exageraciones, el destino del mundo puede depender a veces de la capacidad para hacer ciencia incluso de lo imposible. Pues bien, las condiciones, hoy, para pensar lo imposible, y para trabajar con ello son: la imaginación, la fantasía, las pompas de intuición, algunas de las lógicas no-clásicas, la simulación, y el uso de grandes bases de datos, y por tanto la analítica de datos. Como se aprecia, estas son unas conclusiones abiertas. La explicación detallada de la Tabla 2 constituye el objeto de otro texto aparte.

\section{Bibliografía}

Couloubaritsis, L. 2014. La philosophie face à la question de la complextité. Paris: Éditions OUSIA.

Prigogine I. y Stengers, I. 2002. La mueva alianza: La nueva metamorfosis de la ciencia. Madrid: Alianza Editorial.

Watson, P. 2017. Convergencias. El orden subyacente en el corazón de la ciencia. Barcelona: Crítica.

Recibido el 27 Dic 2018

Aceptado el 13 Feb 2019 